\title{
Belle: CPV with Kpipi/pipipi(rho pi)
}

\author{
Jasna Dragic ${ }^{* \dagger}$ \\ KEK, Japan \\ E-mail: jasna@bmail.kek.jp
}

We report results on studies of $C P$ violation in the three-body charmless decay $B^{ \pm} \rightarrow K^{ \pm} \pi^{ \pm} \pi^{\mp}$ and $B^{0} \rightarrow \pi^{+} \pi^{-} \pi^{0}\left(\rho^{0} \pi^{0}\right)$. We find evidence at the $3.9 \sigma$ level for large direct $C P$ violation in the decay $B^{ \pm} \rightarrow \rho(770)^{0} K^{ \pm}$. This is the first evidence for $C P$ violation in a charged meson decay. We also find evidence of $B^{0} \rightarrow \rho^{0} \pi^{0}$ decay and perform the first measurement of the direct $C P$ violating asymmetry in this mode. The measurements are based on a data sample that contains 386 million $B \bar{B}$ pairs collected with the Belle detector at the KEKB asymmetric-energy $e^{+} e^{-}$(3.5 on $8.0 \mathrm{GeV})$ collider. KEKB operates at the $\Upsilon(4 S)$ resonance $(\sqrt{s}=10.58 \mathrm{GeV})$ with a peak luminosity that exceeds $1.5 \times 10^{34} \mathrm{~cm}^{-2} \mathrm{~s}^{-1}$.

International Europhysics Conference on High Energy Physics

July 21st - 27th 2005

Lisboa, Portugal

* Speaker.

${ }^{\dagger}$ For the Belle Collaboration 


\section{Introduction}

In decays of $B$ mesons to two-body final states ( $B \rightarrow K \pi, \pi \pi$, etc), direct $C P$ violation (DCPV) can only be observed as a difference in $B$ and $\bar{B}$ decay rates. In decays to three-body final states which are often dominated by quasi-two-body channels, DCPV can also manifest itself as a difference in relative phase between two quasi-two-body amplitudes that can be measured via amplitude (Dalitz) analysis. Although DCPV has been observed in decays of neutral $K$ mesons [1] and recently in neutral $B$ meson decays [2] no $C P$ violation in decays of charged mesons has been found to date.

Recently, the time-dependent amplitude analysis of $B^{0} \rightarrow \pi^{+} \pi^{-} \pi^{0}$ decays, which can be used to extract the Unitarity triangle angle $\phi_{2}$, has been performed for the first time [3]. In these studies the $B^{0} \rightarrow \rho^{0} \pi^{0}$ contribution is assumed to be small. However, improved evidence for this decay would suggest that a less simplified Dalitz-plot analysis is necessary. Further, $B^{0} \rightarrow \rho^{0} \pi^{0}$ branching fraction along with its $C P$ asymmetry are needed to complete the $B^{+/ 0} \rightarrow \rho \pi$ isospin pentagon for the isospin analysis method of extracting $\phi_{2}$.

\section{Data Sample \& Event Selection}

Analysis of DCPV in the three-body charmless $B^{ \pm} \rightarrow K^{ \pm} \pi^{ \pm} \pi^{\mp}$ decay is performed by means of Dalitz analysis technique [4]. Measurements of $B^{0} \rightarrow \rho^{0} \pi^{0}$ are performed in the $\rho^{0}$-dominated region of $B^{0} \rightarrow \pi^{+} \pi^{-} \pi^{0}$ phase-space [5]. Both analyses are based on a data sample that contains $386 \pm 5$ million $B \bar{B}$ pairs, collected with the Belle detector at the KEKB collider. The presented measurements supersede the results reported in Ref. [6] and [7].

We identify $B$ candidates with two kinematic variables: $\Delta E=\left(\sum_{i} \sqrt{c^{2} \mathbf{p}_{i}^{2}+c^{4} m_{i}^{2}}\right)-E_{\text {beam }}^{*}$ and $M_{\mathrm{bc}}=\frac{1}{c^{2}} \sqrt{E_{\text {beam }}^{* 2}-c^{2}\left(\sum_{i} \mathbf{p}_{i}\right)^{2}}$, where the summation is over all particles from a $B$ candidate; $\mathbf{p}_{i}$ and $m_{i}$ are their c.m. three-momenta and masses, respectively. The dominant background to the charmless three-body decays comes from continuum events, $e^{+} e^{-} \rightarrow q \bar{q}(q=u, d, s, c)$. This type of background is mostly suppressed using variables that characterize the event topology. For the $B^{0} \rightarrow \rho^{0} \pi^{0}$ mode, additional suppression is achieved through use of the Belle flavour tagging parameter $r$ [8], which can be used as a measure of the confidence that the remaining particles in the event (other than $\pi^{+} \pi^{-} \pi^{0}$ ) originate from a flavour specific $B$ meson decay.

Backgrounds from $B$ decays are identified using large Monte Carlo (MC) simulated samples. Possible contributions to the $K^{+} \pi^{+} \pi^{-} / \pi^{+} \pi^{-} \pi^{0}$ final state from charmed $(b \rightarrow c)$ backgrounds are explicitly vetoed for decays $B \rightarrow D h$ and $B \rightarrow J / \psi(\psi(2 S))\left[\mu^{+} \mu^{-}\right] h$, where $h$ stands for a charged pion or kaon, by applying requirements on the invariant mass of the appropriate twoparticle combination. The remaining $b \rightarrow c$ combinatorial background is taken into account when fitting the data. The most significant background from charmless $B$ decays to $B^{ \pm} \rightarrow K^{ \pm} \pi^{ \pm} \pi^{\mp}\left(B^{0} \rightarrow\right.$ $\left.\rho^{0} \pi^{0}\right)$ originates from $B^{+} \rightarrow \eta^{\prime} K^{+}, B^{+} \rightarrow \pi^{+} \pi^{+} \pi^{-}$and $B^{0} \rightarrow K^{+} \pi^{-}\left(B^{+} \rightarrow \rho^{+} \rho^{0}, B^{+} \rightarrow \rho^{+} \pi^{0}\right.$ and $\left.B^{+} \rightarrow \pi^{+} \pi^{0}\right)$. These backgrounds cannot be removed and are included in the data fit. The $\Delta E\left(M_{\mathrm{bc}}\right)$ distributions for $B^{ \pm} \rightarrow K^{ \pm} \pi^{ \pm} \pi^{\mp}$ and $B^{0} \rightarrow \rho^{0} \pi^{0}$ candidates that pass all the selection requirements are shown in Fig. 1 (a) and Fig. 2 (a, b) respectively. A detailed description of the event selection and background suppression techniques used in $B^{ \pm} \rightarrow K^{ \pm} \pi^{ \pm} \pi^{\mp}$ and $B^{0} \rightarrow \rho^{0} \pi^{0}$ analyses can be found in Ref. [4] and [5], respectively. 

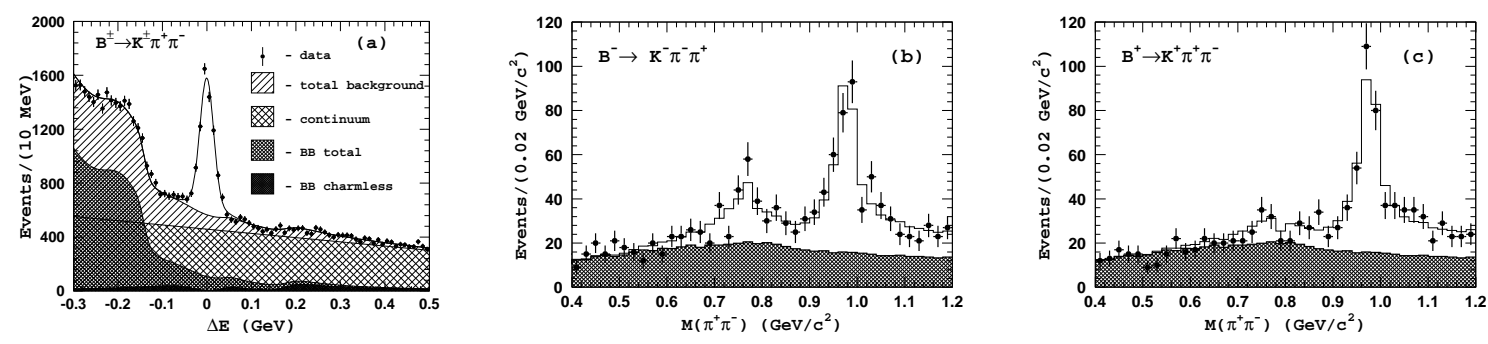

Figure 1: (a) $\Delta E$ distribution for $B^{ \pm} \rightarrow K^{ \pm} \pi^{ \pm} \pi^{\mp}$ candidate events; (b), (c) $m_{\pi^{+}} \pi^{-}$mass spectra for $B^{-}$and $B^{+}$events. Points with error bars are data, the open histogram is the fit result and hatched histogram is the background component.

\section{3. $B^{ \pm} \rightarrow K^{ \pm} \pi^{ \pm} \pi^{\mp}$ Dalitz Analysis Results}

Events in the $B$ signal region defined as an ellipse around the $M_{\mathrm{bc}}$ and $\Delta E$ mean values are selected for the amplitude analysis. The $B^{ \pm} \rightarrow K^{ \pm} \pi^{ \pm} \pi^{\mp}$ signal was found to be well described by a coherent sum of $K^{*}(892)^{0} \pi^{+}, K_{0}^{*}(1430)^{0} \pi^{+}, \rho(770)^{0} K^{+}, f_{0}(980) K^{+}, f_{X}(1300) K^{+}, \chi_{c 0} K^{+}$ quasi-two-body channels and a non-resonant amplitude [6]. In this analysis we modify the model by adding two more quasi-two-body channels, $f_{2}(1270) K^{+}$and $\omega(782) K^{+}$, and change the parameterization of the $f_{0}(980)$ lineshape from a standard Breit-Wigner function to a coupled channel Breit-Wigner (Flatté parameterization). For the DCPV search, the amplitude for each quasi-twobody channel, $a e^{i \delta}$, is parameterized such that a plus (minus) sign is allocated to a $B^{+}\left(B^{-}\right)$decay: $a e^{i \delta}\left(1 \pm b e^{i \phi}\right)$. With this parameterization, the $C P$ violating asymmetry $A_{C P}$ for a particular quasitwo-body channel can be calculated as $A_{C P}(f)=\left(N^{-}-N^{+}\right) /\left(N^{-}+N^{+}\right)=-(2 b \cos \varphi) /\left(1+b^{2}\right)$.

To reduce the number of free fit parameters, we fit the data in two steps. First we fix all $b_{i}=0$ (assume no $C P$ violation) and determine the parameters of the $f_{X}(1300), f_{0}(980)$ and the parameter of the non-resonant amplitude. We then repeat the fit to data with these parameters fixed, while $b_{i}$ and $\varphi_{i}$ are allowed to float. We assume no DCPV in $B^{ \pm} \rightarrow \omega(782) K^{ \pm}$and the non-resonant amplitude; possible effects of these assumptions are considered as a part of the model uncertainty.

Table 1: Results of the best fit to $K^{ \pm} \pi^{ \pm} \pi^{\mp}$ events in the $B$ signal region. The first quoted error is statistical and the second is the model dependent uncertainty. The quoted significance is statistical only, calculated as $\sqrt{-2 \ln \left(\mathscr{L}_{0} / \mathscr{L}_{\max }\right)}$, where $\mathscr{L}_{\max }$ and $\mathscr{L}_{0}$ denote the maximum likelihood with nominal fit and with the asymmetry fixed at zero, respectively.

\begin{tabular}{lcccc}
\hline \hline Channel & $b$ & $\varphi($ deg. $)$ & $A_{C P}(\%)$ & Significance $(\sigma)$ \\
\hline \hline$K^{*}(892)^{0} \pi^{ \pm}$ & $0.078 \pm 0.033_{-0.003}^{+0.012}$ & $-18 \pm 44_{-13}^{+5}$ & $-14.9 \pm 6.4_{-0.8}^{+0.8}$ & 2.6 \\
$K_{0}(1430)^{0} \pi^{ \pm}$ & $0.069 \pm 0.031_{-0.008}^{+0.010}$ & $-123 \pm 16_{-5}^{+4}$ & $+7.5 \pm 3.8_{-0.9}^{+2.0}$ & 2.7 \\
$\rho(770)^{0} K^{ \pm}$ & $0.28 \pm 0.11_{-0.09}^{+0.07}$ & $-125 \pm 32_{-85}^{+10}$ & $+30 \pm 11_{-4}^{+11}$ & 3.9 \\
$f_{0}(980) K^{ \pm}$ & $0.30 \pm 0.19_{-0.10}^{+0.05}$ & $-82 \pm 8_{-2}^{+2}$ & $-7.7 \pm 6.5_{-1.6}^{+4.1}$ & 1.6 \\
$f_{2}(1270) K^{ \pm}$ & $0.37 \pm 0.17_{-0.03}^{+0.11}$ & $-24 \pm 29_{-20}^{+14}$ & $-59 \pm 22_{-3}^{+3}$ & 2.7 \\
\hline$\chi_{c 0} K^{ \pm}$ & $0.15 \pm 0.35_{-0.07}^{+0.08}$ & $-77 \pm 94_{-11}^{+154}$ & $-6.5 \pm 19.6_{-1.4}^{+2.9}$ & 0.7 \\
\hline \hline
\end{tabular}

Results of the final fit are given in Table 1. The only channel where the statistical significance of the $C P$ asymmetry exceeds the $3 \sigma$ level is $B^{ \pm} \rightarrow \rho(770)^{0} K^{ \pm}$. Figures $1(\mathrm{~b}, \mathrm{c})$ show the $\pi^{+} \pi^{-}$ invariant mass distributions for the $\rho(770)^{0}-f_{0}(980)$ mass region separately for $B^{-}$and $B^{+}$events. 


\section{4. $B^{0} \rightarrow \rho^{0} \pi^{0}$ Results}

We measure the signal yield using an extended unbinned maximum-likelihood fit to the $\Delta E$ $M_{\mathrm{bc}}$ distribution. We obtain $51_{-13}^{+14}$ signal events with a significance of $4.2 \sigma$ including systematic uncertainties and measure the branching fraction to be $\mathscr{B}\left(\rho^{0} \pi^{0}\right)=\left(3.12_{-0.82}^{+0.88}(\text { stat })_{-0.76}^{+0.60}(\right.$ syst $\left.)\right) \times$ $10^{-6}$. In order to check that the signal candidates originate from $B^{0} \rightarrow \rho^{0} \pi^{0}$ decays, we change the criteria on $m_{\pi^{+} \pi^{-}}$and $\cos \theta_{\text {hel }}^{\rho}$ in turn, and repeat fits to the $\Delta E-M_{\mathrm{bc}}$ distribution. The yields obtained in each $m_{\pi^{+} \pi^{-}}$and $\cos \theta_{\text {hel }}^{\rho}$ bin are shown in Fig. 2 (c) and (d).

Having observed a significant $B^{0} \rightarrow \rho^{0} \pi^{0}$ signal, we utilize the $B^{0} / \bar{B}^{0}$ separation provided by the flavour tagging to measure the $C P$ asymmetry by defining the probability density function (PDF) as: $\mathscr{P}_{j, l}^{i}=\frac{1}{2}\left[1+q^{i} \cdot\left(A_{\mathrm{CP}}^{\prime}\right)_{j, l}\right] P_{j, l}\left(M_{\mathrm{bc}}^{i}, \Delta E^{i}\right)$ where $q$ is the b-flavour charge $[q=+1(-1)$ when the tagging $B$ meson is a $\left.B^{0}\left(\bar{B}^{0}\right)\right], P_{j, l}$ is the two dimensional PDF in $\Delta E$ and $M_{\mathrm{bc}}$, and $A_{\mathrm{CP}}^{\prime}$ is the effective charge asymmetry, such that $\left(A_{\mathrm{CP}}^{\prime}\right)_{j, l}=\left(A_{\mathrm{CP}}\right)_{j}\left(1-2 \chi_{d}\right)\left(1-2 w_{l}\right)$. Here, $\left(A_{\mathrm{CP}}\right)_{j}$ are the charge asymmetries for the signal and the background components, $\chi_{d}$ is the time-integrated mixing parameter and $w_{l}$ is the wrong-tag fraction. The only free $A_{\mathrm{CP}}$ parameter in the nominal fit, is that of our signal; the rest are fixed to be zero. We measure the direct $C P$ asymmetry of $B^{0} \rightarrow \rho^{0} \pi^{0}$ to be $A_{C P}=-0.49_{-0.81}^{+0.67}$ (stat $)_{-0.24}^{+0.20}$ (syst). To illustrate the asymmetry, the results are shown separately for $\rho^{0} \pi^{0}$ candidate events tagged as $B^{0}$ and $\bar{B}^{0}$ in Fig. 2 (e, f).
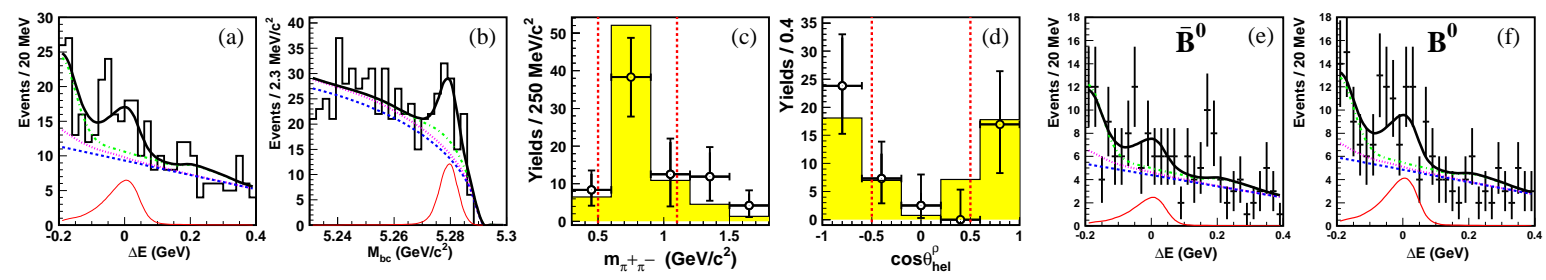

Figure 2: (a), (b) Distribution of $\Delta E\left(M_{\mathrm{bc}}\right)$ in the signal region of $M_{\mathrm{bc}}(\Delta E)$. Projection of the fit result is shown as the thick solid curve; the thin solid line represents the signal component; the dashed, dotted and dash-dotted curves represent, respectively, the cumulative background components from continuum processes, $b \rightarrow c$ decays, and charmless $B$ backgrounds. (c), (d) Distributions of fit yields in $m_{\pi^{+}} \pi^{-}$and $\cos \theta_{\text {hel }}^{\rho}$ variables for $\rho^{0} \pi^{0}$ candidate events. Points with error bars represent data fit results, and the histograms show signal MC expectation. (e) (f) $\Delta E$ distributions shown separately for events tagged as $\bar{B}^{0} / B^{0}$

\section{References}

[1] J.R. Batley et al. (NA48 Collab.), Phys. Lett. B544, 97 (2002); A. Alavi-Harati et al. (KTeV Collab.), Phys. Rev. D67, 012005 (2003).

[2] B. Aubert et al. (BaBar Collab.), Phys. Rev. Lett. 93, 131801 (2004); Y. Chao et al. (Belle Collab.), Phys. Rev. Lett. 93, 191802 (2004).

[3] B. Aubert et al. (BaBar Collab.), hep-ex/ 0408099 , submitted to the ICHEP 04 conference.

[4] K. Abe et al. (Belle Collab.), hep-ex/0509001 and references therein.

[5] K. Abe et al. (Belle Collab.), hep-ex/0508007 and references therein.

[6] A. Garmash et al. (Belle Collab.), Phys. Rev. D71, 092003 (2005).

[7] J. Dragic et al. (Belle Collab.), Phys. Rev. Lett. 93, 131802 (2004).

[8] H. Kakuno et al., Nucl. Instr. and Meth. A 533, 516 (2004). 